\title{
Design and Realization of a Certain Type of Information Equipment Master Circuit Board Testing and Training Platform
}

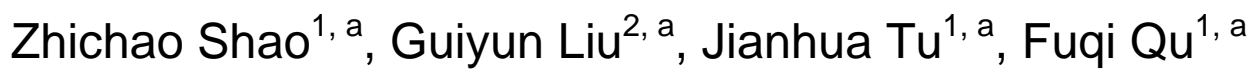 \\ ${ }^{1}$ Wuhan Mechanical Technology College, WuHan, 430075, China \\ ${ }^{2}$ Wuhan University of technology huaxia college, WuHan, 430223, China \\ aemail: shaozhichao@sina.com
}

Keywords: Master Circuit Board; Testing and Training Platform; adapter; Teaching Software.

\begin{abstract}
A certain type of command and control system testing and training platform is designed in order to realize clear display of the operation, implement of the capability teaching, testing and fault diagnoses conveniently. This thesis which is a part of the program introduces the design and realization of a certain type of information equipment master circuit board testing and training platform. The platform consists of adapter and teaching software. Experiments indicate that the platform is reliable in the operation, can improve the efficiency of circuit board teaching and provide a method for circuit board testing and fault diagnoses.
\end{abstract}

\section{Introduction}

A certain type of radio is a high-tech electronic information equipment with the characteristics of complex structures and expensive cost, which is widely used in our army's various command and control systems. At present, there is a lot of inconvenience in the teaching of working principles of the information equipment, especially circuit working principles. Only when the information equipment is taken apart can it show its internal structure. Due to the internal circuit boards of the information equipment are designed in sub-block and packaged respectively, and the circuit boards must be encapsulated when working with charge, there is no way to implement the live signal testing and analysis with electricity to key test points of the circuit board of the information equipment. To solve this problem, a testing and training platform of a certain type of command system is developed which can solve the difficulties described above, and implement the teaching of the circuit operating principle of a certain type of information equipment printed circuit board (PCB) level and fault diagnosis visually.

\section{Platform Introduction}

The hardware components of a certain type command system testing and training platform can exert excitation signal to the circuit board when the printed circuit board waiting be tested is from the individual equipment. The platform creates the working environment, which ensures the circuit board can work in normal state, measures actually key points signal of the circuit board by universal testing equipments, and also by the host computer to display the working principle of PCB, signal flow, as well as the theoretical value of key points signal. Through the combination of hardware and software, the platform developed a variety of adapters and teaching software for different types of printed circuit boards.

\section{Principle of the information equipment master circuit board}

The information equipment includes many types and circuit board. This thesis which takes example for master circuit board introduces the design and realization of a certain type of information equipment testing and training platform. The function of information equipment is realized mainly by master circuit board. The information equipment master circuit board is composed of CPU, keyboard and display circuit board, serial communication circuit board and control switch circuit board. CPU is DS80C320 singlechip which is produced by DALLAS 
corporation. The keyboard and display circuit board is input and output equipment of the information equipment. The serial communication circuit board realize voltage switch by using RS-232C voltage switch CMOS chip.The serial interface TXD and RXD of double serial interface DS80C320 singlechip realize communication with Radio as wireless serial interface. The information equipment master circuit board schematic framework diagram is shown in Figure 1.

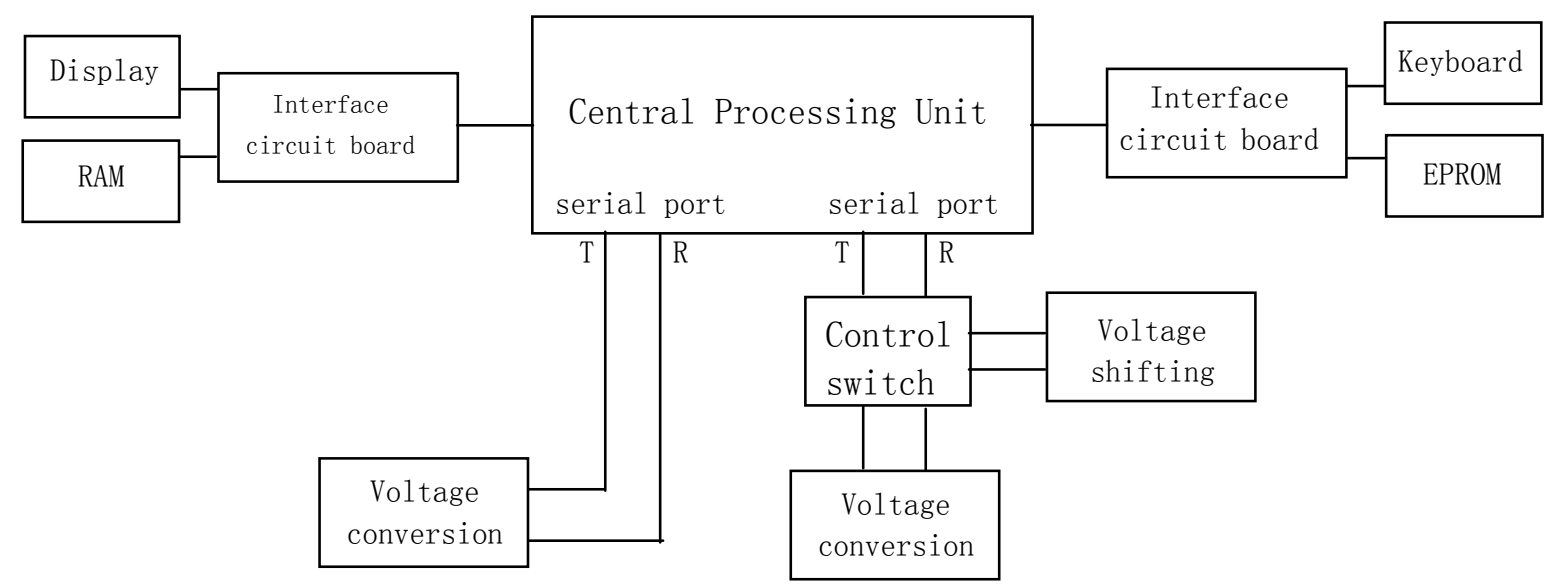

Fig.1. The information equipment master circuit board schematic framework diagram

\section{Platform design and implementation}

The testing and training platform is composed of undetected PCB, PCB adapter, the host computer, as well as general testing equipment. The structure of the command system PCB general testing and training platform is shown in Figure 2.

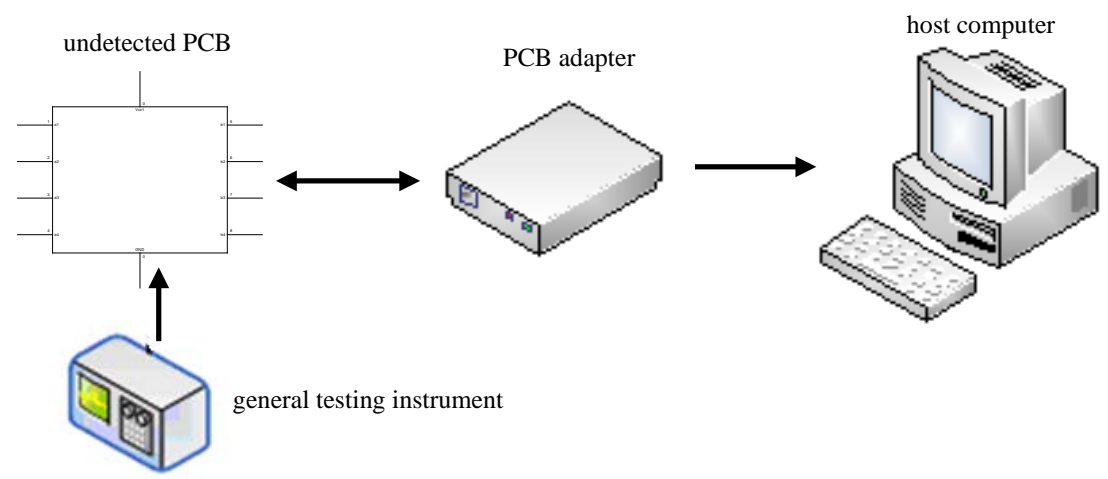

Fig.2. The structure of a certain command system PCB general testing and training platform

The platform will develop corresponding PCB adapters for all types PCB. The adapter, by receiving commands from the host computer serial port, provides the required power input and excitation input to the undetected PCB, and then after receiving the signal output from the undetected PCB to sent the output signal to the host computer through the serial port eventually. Running on the host computer, the testing and training software can control the adapter to generate different excitation signals to the undetected PCB; after the host computer receives the feedback signal from the adapter, the test results will be displayed on the monitor in the form of graph. At the same time, the software system is able to demonstrate all the key functional requirements, operating principles of the PCB and the structure of the equipments which they belong to. The general testing instrument, which consists of multimeters, oscilloscopes etc, is used to test the actual signals of the critical detection point of the PCB. Besides, the platform is also equipped with a DC power supply, excitation sources like the signal generator. 
- Hardware Design of the Platform

The platform hardware provides the circuit boards with corresponding adapters, in accordance with the specific circumstances of the PCB, different adapters will be developed to meet the different working states of the PCB. Through receiving the commands sent by the host computer serial port, the adapter sets out to adjust the working condition, provides a variety of excitation signal to the undetected PCB, and at the same time sends commands to the host computer through the serial port. After the adapter controls the working status of the circuit board and the PCB works properly, the same test results(theoretical value) with the host computer software will be achieved by using the general testing instrument to test the measured values of the key points of the circuit board so as to achieve the testing and training function of the PCB. A certain type of information equipment master circuit board adapter design diagram is shown in Figure 3.

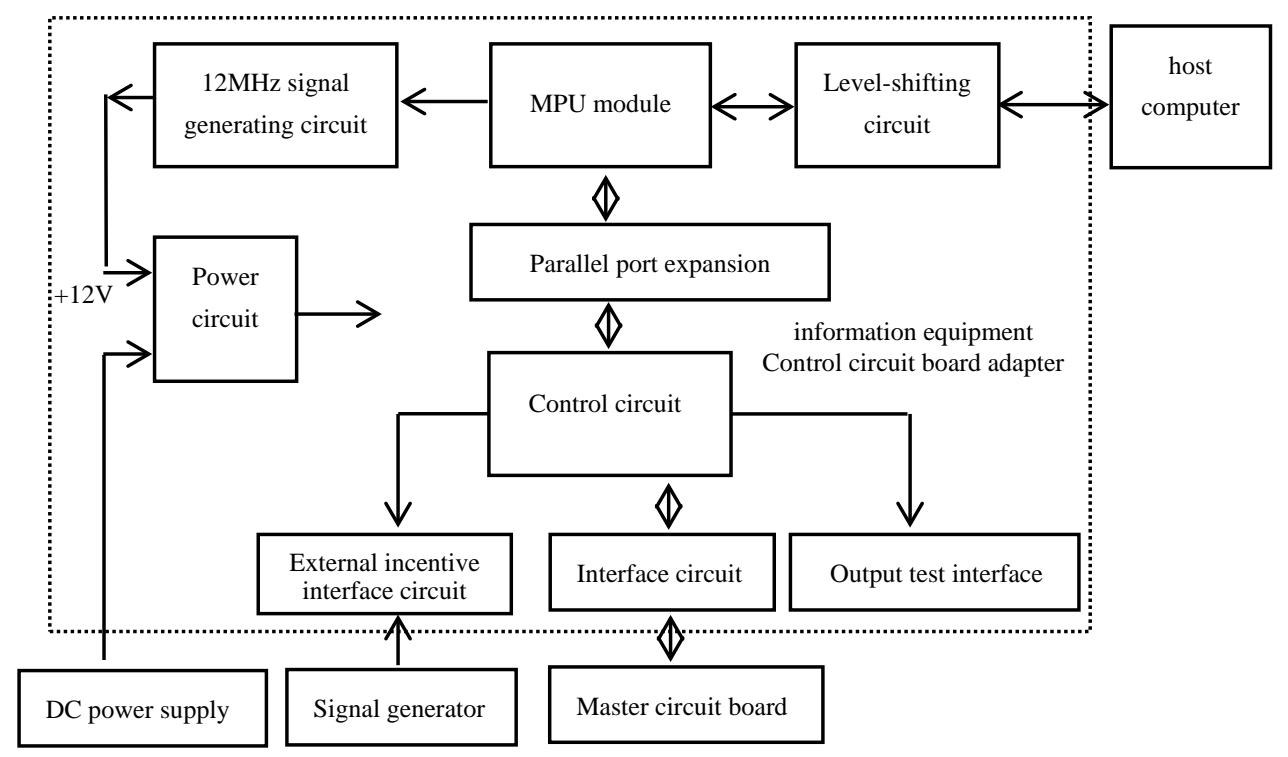

Fig.3. Information equipment master circuit board adapter structure

- Software Design of the Platform

The PCB testing and training software is the software proportion of the host computer in the testing and training system, which is used to display the structure of the PCB, operating principles, schematics, circuit board and the key point signal theory waveform. After selecting the PCB with specific model of equipment, the testing and training software will send instructions to the PCB through the host computer serial port adapter. And then the adapter will generate various excitation signals required in the running of PCB to further controls and adjusts the working status of the circuit board.

The signal testing function of the circuit critical points in the testing and training software is realized through the following programs: increase the critical points in the circuit diagram, corresponding theoretical value waveform is pre-stored in the corresponding critical points, then click the key point and the teaching software interface can display the pre-stored signal waveform on the host computer.

Each adapter corresponds to one or more PCB, each PCB corresponds to its circuit diagram and the main interface of teaching software which consists of PCB digital real photos, digital schematic of the PCB and PCB critical detection point signal theoretical waveforms. The platform developing software chooses VB, the teaching software operating results interface is shown in Figure 4, the software interface can display the circuit schematics, circuit board photos, the specific location of critical detection points in the circuit board and the detection point signal waveform simultaneously. Users can choose one from the adapters in using, and then select one PCB from the specific adapters. The teaching software communicates with PCB adapter via the host computer serial port, then sends commands to the adapter through the serial port. The commands are not only the basis of 
the adapter to generate the corresponding excitation signal, but also can receive the command sent back through the serial port adapter.

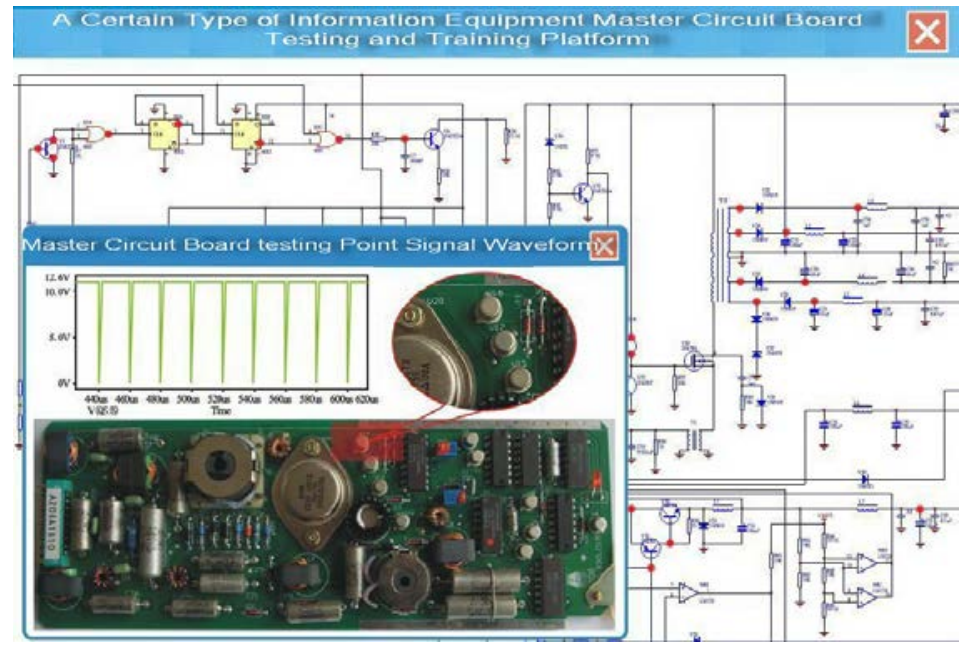

Fig.4. Interface diagram of the testing and training software

\section{Summary}

By using the adapter the testing and training platform provide a work environment for the PCB, which can not only make the information equipment master circuit board work properly without from the information equipment itself, but also show perfectly the structure of information equipment internal circuit board and its working principle, at the same time it completes testing, teaching, the task of testing and fault diagnosis of the information equipment circuit board. It greatly facilitates the mounting of teaching and fault detection and maintenance, overcomes the difficulties of non-intuitive and unpicturesque display in traditional mounting instruction mode which eliminates the unnecessary work of complex disassembly and test equipment connections. Through software and hardware environmental testing, the platform runs stably and reliably, which can greatly improve the efficiency and level of implementation in teaching, as well as provide a convenience for the actual test and maintenance.

Further work of the research is to optimize software system and improve stability of platform, to make the message box more graphical and simplify the work of operating staff for a greater efficiency.

\section{References}

[1]Liu Cun, Modern Detection Techniques, Beijing: Mechanical Industry Press, 2005, pp.65-69.

[2]Yu Mengchang.Fundamentals of Digital Electronics Concise Guide[M] Beijing: Higher Education Press ,1999:98 -104.

[3]Zhou Yuwei, Automatic Detection Technology, Wuhan: Huazhong University of Science Press ,1995, pp.112 -116.

[4]Houbo Heng, Libo Cheng, 16 Microcomputer Principle and Interface Technology. Xi'an: Electronic Science and Technology University Press ,1996, pp.79 -83.

[5]LI Hai-ou, ZHANG Zhong-ting, JIANG Shou-da,A research of general testing software based on VXI bus. High Technology Letters,2000,1(2);75-77. 